\title{
Bosonic superfluid transport in a quantum point contact
}

\author{
Shun Uchino $\odot^{1, *}$ and Jean-Philippe Brantut ${ }^{2}$ \\ ${ }^{1}$ Weseda Institute for Advanced Study, Waseda University, Shinjuku, Tokyo 169-0051, Japan \\ ${ }^{2}$ Institute of Physics, EPFL, 1015 Lausanne, Switzerland
}

(Received 4 July 2019; revised manuscript received 23 February 2020; accepted 13 May 2020; published 4 June 2020)

\begin{abstract}
We present a microscopic theory of heat and particle transport of an interacting, low-temperature BoseEinstein condensate in a quantum point contact. We show that, in contrast to charged, fermionic superconductors, bosonic systems feature tunneling processes of condensate elements, leading to the presence of odd-order harmonics in the AC Josephson current. A crucial role is played by an anomalous tunneling process where condensate elements are coherently converted into phonon excitations, leading to even-order harmonics in the $\mathrm{AC}$ currents as well as a DC contribution. At a low bias, we find dissipative components obeying Ohm's law and bias-independent nondissipative components, in sharp contrast to fermionic superconductors. Analyzing the DC contribution, we find zero thermopower and Lorenz number at zero temperature, a breakdown of the bosonic Wiedemann-Franz law. These results highlight the importance of the anomalous tunneling process inherent to charge-neutral superfluids. The consequences could readily be observed in existing cold-atom transport setups.
\end{abstract}

DOI: 10.1103/PhysRevResearch.2.023284

\section{INTRODUCTION}

A mesoscopic system connected to reservoirs is one of the most important examples of nonequilibrium quantum statistical physics [1]. For a long time, this system was mainly discussed in electron systems, which revealed nontrivial outcomes absent in bulk systems such as conductance quantization [2], current noise [3], and fluctuation relations [4]. Recently, atomic quantum gases have emerged as an alternative route to the investigation of mesoscopic systems $[5,6]$, in particular, single-mode quantum point contacts (QPCs) [7]. QPCs are the cornerstone of mesoscopic quantum devices, as building blocks for quantum coherent devices such as quantum dots and interferometers. Their counterparts in atomic gases open the perspective of complex, quantum coherent 'atom-tronic' devices [8-11] featuring controlled interactions [12] and the possibility of using fermionic or bosonic statistics [13-15].

In contrast with superconductors, which are charged, atomic quantum gases are charge neutral. As a result, in the superfluid phase, there always exist gapless collective modes which are not present in charged systems due to the AndersonHiggs mechanism $[16,17]$. The fact that the dominant lowenergy excitations of superconductors are of the pair-breaking type has spectacular consequences such as multiple Andreev reflections in superconductor-normal interfaces [18] or QPCs [19], yet little is known concerning the role played by gapless collective excitations in quantum transport in charge-neutral

\footnotetext{
*shun.uchino@aoni.waseda.jp

Published by the American Physical Society under the terms of the Creative Commons Attribution 4.0 International license. Further distribution of this work must maintain attribution to the author(s) and the published article's title, journal citation, and DOI.
}

superfluids. In the case of a Bose-Einstein condensate (BEC), relevant to two-terminal systems of cold atoms [6,20], as well as superfluid helium in nanopores [21], a hydrodynamic description correctly predicts the Josephson dynamics [22-25] and dissipation associated with topological excitations in wide junctions but fails in the case of a QPC.

In this paper, we present a microscopic theory of lowtemperature transport of interacting neutral bosons through a single-mode QPC. Modeling the system with the tunneling Hamiltonian [26-31] and the Bogoliubov theory of interacting condensates [32,33], we use the Keldysh formalism [34,35] to derive a current formula applicable to heat and particle transport up to a nonlinear response regime. We elucidate the crucial role of bosonic enhancement and gapless Bogoliubov modes in superfluid transport, which is revealed in the differences between odd and even harmonics of the AC Josephson current.

We predict dissipative components obeying Ohm's law even at zero temperature with a fully superfluid system, which is in sharp contrast with the case of charged superconductors [26]. Then we consider the DC transport of heat and particle currents, which, in spite of obeying Ohm's law [36], shows a large breakdown of the bosonic Wiedemann-Franz law. Finally, we propose experiments to confirm our findings in existing two-terminal setups of ultracold atomic gases.

This paper is organized as follows. In Sec. II, we present the model and methods yielding an expression for the mesoscopic current, which is the basis of this work. In Sec. III we use this expression to obtain results for the AC and DC components of the current at fixed biases. In Sec. IV, we present predictions for cold-atom experiments, including the effect of the trapping potential, for the time evolution of thermodynamic quantities and Shapiro resonances. Section V summarizes our results and mentions possible applications of this work. In Appendixes A and B, some technical details on the theoretical analyses are provided. 


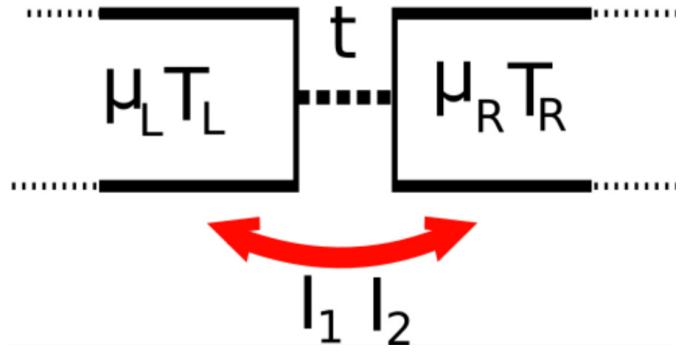

FIG. 1. Model used for the calculation, with $T_{i}$ and $\mu_{i}$ the temperature and chemical potential in reservoir $i, t$ the transmission amplitude for single atom across the contact, and $I_{1}$ and $I_{2}$ the particle and heat currents, respectively.

\section{MODEL AND METHODS}

We consider a two-terminal transport system, with two macroscopic reservoirs [37] filled with a weakly interacting BEC and connected by a mesoscopic channel, as shown in Fig. 1. We describe the channel as a single-mode QPC, which is adequate when its width and length are much shorter than the coherence length of the BEC. In this case, the details of the motion of atoms in the constriction become irrelevant [19,38], which allows one to start with the tunneling Hamiltonian $[26-31,39,40]$

$$
H=K_{L}+K_{R}+t\left[\psi_{L}^{\dagger}(\mathbf{0}) \psi_{R}(\mathbf{0})+\psi_{R}^{\dagger}(\mathbf{0}) \psi_{L}(\mathbf{0})\right],
$$

where $\psi_{L(R)}(\mathbf{x})$ and $K_{L(R)}$ are the field operator of the reservoir $L(R)$ and the grand-canonical Hamiltonian of a Bose gas $\left[K_{L(R)}=H_{L(R)}-\mu_{L(R)} N_{L(R)}\right.$ with the Hamiltonian operator $H_{L(R)}$ and with the number operator $N_{L(R)}$ ], respectively. The last two terms in Eq. (1) describe exchanges of particles between the reservoirs, where $t$ is the tunneling amplitude. In the two-terminal system, the particle and heat current operators can be expressed as $I_{1}=-\dot{N}_{L}$ and $I_{2}=-\dot{K}_{L}$, respectively.

We consider a regime of weak interaction and high degeneracy and describe the BECs in the reservoirs with the Bogoliubov theory $[32,33]$. To construct a theory of heat and particle transport, we adopt the Keldysh formalism [34,35], allowing for a description of the dynamics in the presence of an arbitrary bias and tunneling amplitude. Under a fixed chemical potential bias $(\Delta \mu)$ and temperature bias $(\Delta T)$, the particle and heat currents at time $\tau$ can be expanded as a Fourier series in harmonics of $\Delta \mu$ and be obtained as (see Appendix A for the derivation)

$$
\begin{aligned}
& I_{q}(\tau)= \operatorname{Re}\left[\sum_{m \in \mathbb{Z}} I_{q, 2 m-1} e^{(2 m-1) i \phi(\tau)}+\sum_{m \in \mathbb{Z}} I_{q, 2 m} e^{2 m i \phi(\tau)}\right] \\
& \equiv I_{q}^{\text {odd }}(\tau)+I_{q}^{\text {even }}(\tau), \\
& I_{q}^{\text {odd }}=2 \operatorname{Re}\left[\left(\left\{i \partial_{\tau}-\Delta \mu\right\}^{q-1} \hat{T}_{R L}^{R}\right) \circ \hat{g}_{L R}^{<} \circ \hat{T}_{R L}^{A} \hat{t}^{-1}\right. \\
&\left.\quad+i \hat{t}\left(\partial_{\tau}^{q-1} \hat{g}_{L L}^{R}\right) \circ \hat{T}_{L R}^{R} \circ \hat{g}_{R L}^{<} \circ \hat{T}_{L R}^{A} \circ \hat{g}_{R R}^{A}\right]_{11}, \\
& I_{q}^{\text {even }}=2 \operatorname{Re}\left[i\left(\partial_{\tau}^{q-1} \hat{g}_{L L}^{R}\right) \circ \hat{T}_{L R}^{R} \circ \hat{g}_{R R}^{<} \circ \hat{T}_{R L}^{A}\right. \\
&\left.\quad+\left(\left\{i \partial_{\tau}-\Delta \mu\right\}^{q-1} \hat{T}_{R L}^{R}\right) \circ \hat{g}_{L L}^{<} \circ \hat{T}_{L R}^{A} \circ \hat{g}_{R R}^{A}\right]_{11},
\end{aligned}
$$

where $q=1$ or $2, \partial_{\tau} \phi(\tau)=\Delta \mu$, and $\circ$ represents integration over the internal time variable from $-\infty$ to $+\infty$. We note that in this paper we set $\hbar=k_{B}=1$ except for plots in figures. In addition, $\hat{g}^{R}, \hat{g}^{A}$, and $\hat{g}^{<}$are the uncoupled retarded, advanced, and lesser Green's functions, which have $2 \times 2$ structures conventionally employed in a BEC system [34,41]. The Keldysh formalism incorporates the coherent sum of all processes by which atoms can traverse the channel. Such a beyond-linearresponse effect is accumulated in the renormalized hopping matrix $\hat{T}^{R(A)}$ defined as

$$
\hat{T}_{R L}^{R(A)}=\hat{t}+\hat{t} \circ \hat{g}_{L L}^{R(A)} \circ \hat{t}^{\dagger} \circ \hat{g}_{R R}^{R(A)} \circ \hat{T}_{R L}^{R(A)},
$$

where $\hat{T}_{L R}^{R(A)}\left(\tau, \tau^{\prime}\right)=\left[\hat{T}_{R L}^{A(R)}\left(\tau^{\prime}, \tau\right)\right]^{\dagger} \quad$ and $\quad \hat{t}\left(\tau, \tau^{\prime}\right)=$ $\operatorname{diag}\left(t e^{-i \phi(\tau)}, t e^{i \phi(\tau)}\right) \delta\left(\tau-\tau^{\prime}\right)$. Although the structure is similar to that of superconducting QPCs, in that the reservoir Green's functions are $2 \times 2$ matrices, the emergence of $I_{q}^{\text {odd }}$ is peculiar to the bosonic system. In fermionic systems $\hat{g}_{L R(R L)}^{<}=\hat{0}$ and odd harmonics are absent, because fermionic superfluidity involves pairing. The current expression obtained above is the basis for the analysis of mesoscopic BEC systems. In the weak-tunnel-coupling regime, it describes, in particular, a BEC in a double-well [22-24] as long as motion along the finite extension of the junction can be neglected.

\section{RESULTS}

In this section we consider the situation in which the chemical potential in the reservoirs is fixed, which is standard in the condensed-matter physics context. In order to connect to quantum gas experiments, in what follows we present figures for which an average chemical potential $\mu=400 \mathrm{nK}$, the scattering length $a=25 \mathrm{~nm}$, and the average density in the reservoirs $n=10^{19} \mathrm{~m}^{-3}$.

\section{A. AC component}

The first outcome of our formalism is the structure of the AC components of the particle current at zero temperature. Due to bosonic enhancement, the dominant transport process is associated with the condensate elements. In the Bogoliubov prescription [41], the field operator is decomposed into the the c-number part, describing the condensate, and the rest, describing the noncondensate elements, and so is Green's function. In our formulation, such a c-number part (condensate element) appears in the lesser Green's function and is expressed as

$$
\hat{g}_{\alpha \beta}^{<}(\tau) \rightarrow-\frac{i \sqrt{\mu_{\alpha} \mu_{\beta}}}{g}\left(\begin{array}{ll}
1 & 1 \\
1 & 1
\end{array}\right),
$$

where $\alpha, \beta=\mathrm{L}$ or $\mathrm{R}$, and $g$ is the coupling constant of the interatomic interaction [42]. We note that, in contrast to the phonon contribution of the lesser Green's function shown in Appendix A, Eq. (6) does not depend on the Bose distribution function's being a function of the temperature. Thus, the processes associated with the condensate survive at zero temperature.

In the tunneling limit where $\hat{T}_{R L} \rightarrow \hat{t}$, the leading current term is linear in $t$. It arises from the $I_{1,-1}$ component, and the corresponding current is $I_{1}(\tau) \rightarrow-\frac{2 \sqrt{\mu_{L} \mu_{R}} t}{g} \sin \phi(\tau)$. This is the Josephson current associated with the tunneling of 

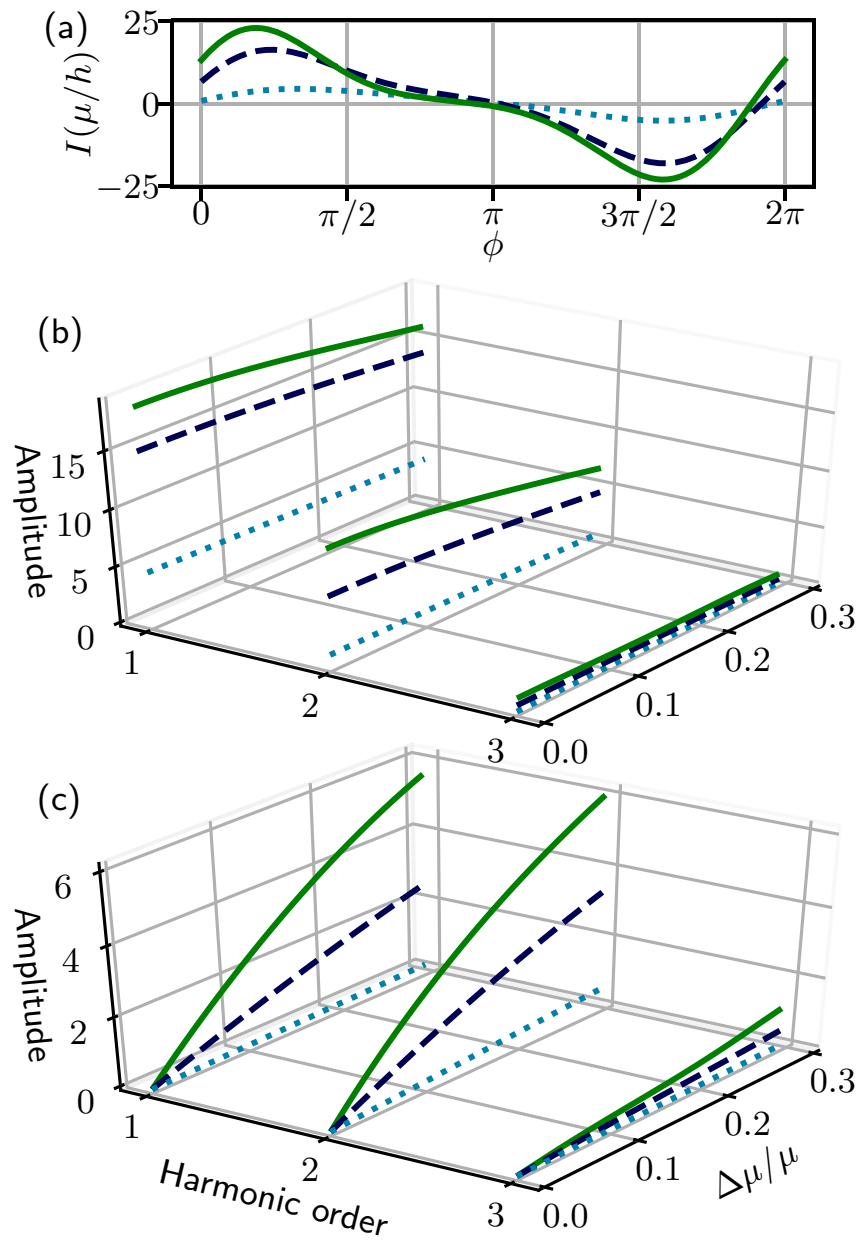

FIG. 2. (a) AC component of the particle current $I_{1}(\phi(\tau))=$ $\sum_{m \neq 0}\left[I_{1, m}^{\mathrm{d}} \cos (m \phi)+I_{1, m}^{\mathrm{nd}} \sin (m \phi)\right] \quad$ for different tunneling amplitudes- $t \rho(\mu)=0.5$ (solid curve), 0.4 (dashed curve), and 0.2 (dotted curve) - for $\Delta \mu / \mu=0.3$. The current is plotted in units of $\mu / h$. (b) The first three components of the nondissipative current amplitude $I_{1, m}^{\text {nd }}$ as a function of $\Delta \mu$. (c) The first three components of the dissipative current amplitude $I_{1, m}^{\mathrm{d}}$.

condensate elements between reservoirs and is consistent with the result in a double-well BEC [22,24,33].

The replacement $\hat{T}_{R L} \rightarrow \hat{t}$ also allows us to discuss the current component quadratic in $t$. This corresponds to the linear response analysis [43] and yields the DC component $I_{1,0}$ and AC components $I_{1, \pm 2}$. An analytic calculation discussed in Appendix B shows the presence of dissipative and nondissipative terms proportional to $\cos 2 \phi(\tau)$ and $\sin 2 \phi(\tau)$, respectively. The dominant microscopic process in even harmonics is the tunneling of a condensate element coherently converted into phonon excitation. This anomalous tunneling process is fundamentally different from the tunneling process of the nondissipative odd harmonics and from tunneling processes in the superconductor case.

To include systematically higher-order tunneling effects with all the virtual processes, we numerically solve Eqs. (2)(5) under the low-energy expression of $\hat{g}^{R(A)}$ shown in Appendix A. Figure 2 depicts the results of the first three AC components for various tunneling amplitudes normalized by
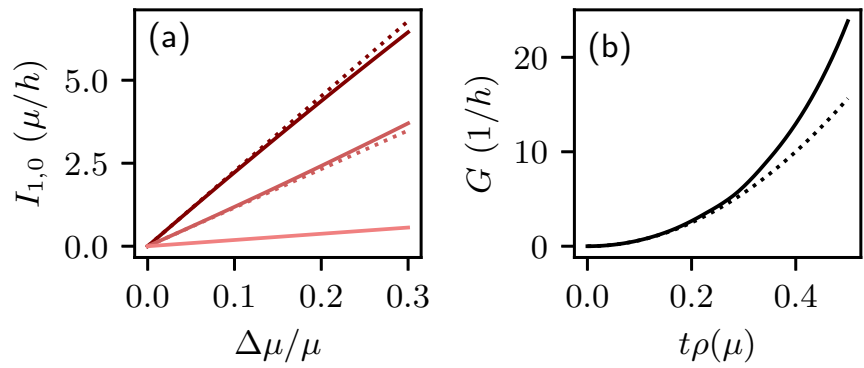

FIG. 3. (a) DC current $I_{1,0}$ as a function of the bias normalized by $\mu$ for different tunneling amplitudes: $t \rho(\mu)=0.5$ (dark-red lines), 0.4 (red lines), and 0.2 (light-red lines). Solid lines show the numerical solution; dashed lines, a linear fit to the data. For $t \rho(\mu)=0.2$ the linear fit is not distinguishable from the exact result. (b) Conductance as a function of tunneling. The solid line shows the exact result; the quadratic dashed line is the result of the linear response theory.

the density of states in the reservoirs at the chemical potential $\rho(\mu)$, as in the case of fermions [26]. As shown in Fig. 2(a), the AC current deviates from sinusoidal behavior with increasing $t$, revealing the important role of higher harmonics.

Moreover, Fig. 2(c) demonstrates the presence of a dissipative term proportional to $\cos \phi(\tau)$, which is absent at the linear response level. Remarkably, our calculation shows that the amplitudes in the dissipative components obey Ohm's law at small $\Delta \mu$, in contrast to the fermionic case, where the nonlinear bias dependence appears [26]. This difference originates from $\hat{g}^{R(A)}(\omega)$, which is nonsingular for bosons but is singular at the pairing gap frequency for fermions [26]. In contrast, we find that the nondissipative component $\propto \sin m \phi(\tau)$ has a nonzero amplitude even at $\Delta \mu=0$ [Fig. 2(c)], comparable with the fermionic case up to nonlinearity [26].

\section{B. DC component}

We now focus on the DC component of the particle current $I_{1,0}$ as a function of $\Delta \mu$ at zero temperature. This is directly measurable in cold-atom experiments where all the $\mathrm{AC}$ components are averaged out [44]. The resulting current-bias relations for different values of $t$ are shown in Fig. 3(a). In all cases, Ohm's law is obeyed in the low-bias regime. This again contrasts with the case of superconductors showing nonlinearities associated with multiple Andreev reflections [19]. This also differs from the case with one-dimensional reservoirs described by the Luttinger liquid, where power-law current-bias relations are obtained due to critical fluctuations [45].

Our formalism allows for a systematic investigation of the conductance in the linear regime as a function of the tunneling amplitude. The result is shown as a solid line in Fig. 3(b). Remarkably, even for moderate tunneling amplitudes, of the order of $0.4 / \rho(\mu)$, the conductance is 10 times larger than that of the upper bound of noninteracting fermions [46]. In the tunneling regime, conductance shows a quadratic dependence on $t$ [dashed line in Fig. 3(b)], suggesting that the linear response theory accurately captures the physics.

To clarify the nature of the DC transport we now include the heat current, focusing again on the linear response regime. An analytic calculation derived in Appendix B shows that 

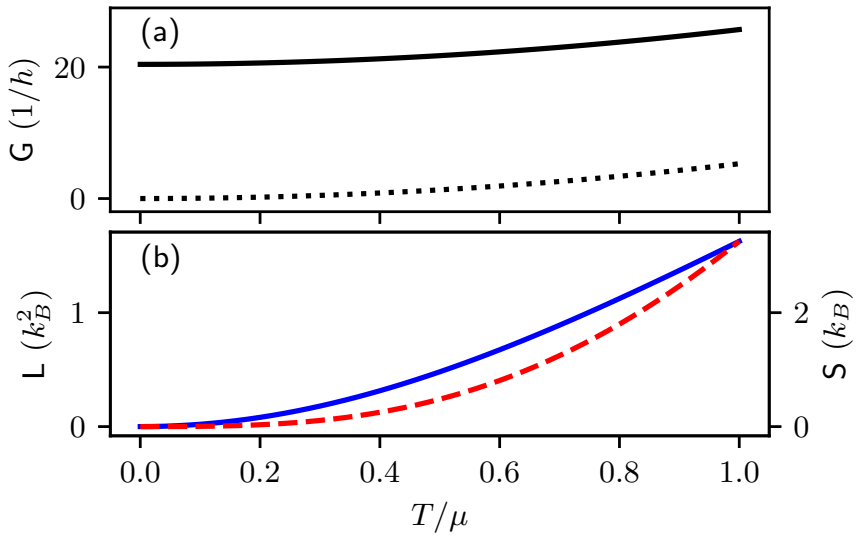

FIG. 4. Temperature dependence of the transport coefficients (see text for the parameters). (a) Conductance including the phonon contributions (solid lines) and excluding them (dashed lines). The latter is consistent with [47]. (b) Lorenz number (solid blue line; left axis) and Seebeck coefficient (dotted red line; right axis), in the tunneling approximation.

the DC particle and heat currents are related to biases by an Onsager matrix:

$$
\left(\begin{array}{l}
I_{1,0} \\
I_{2,0}
\end{array}\right)=\left(\begin{array}{cc}
L_{11} & L_{12} \\
T L_{12} & L_{22}
\end{array}\right)\left(\begin{array}{c}
\Delta \mu \\
\Delta T
\end{array}\right) .
$$

Here, the transport coefficients $\left(L_{11}, L_{12}, L_{22}\right)$ depend on the temperature $T$. They are proportional to $t^{2}$, by hypothesis. The conductance is expressed as $G=L_{11}=\frac{t^{2} \mu^{2}}{\pi c^{3}}\left[\frac{1}{g}+\frac{T^{2}}{12 c^{3}}\right]$ with the speed of sound $c$. It features a zero-temperature contribution of the condensate inversely proportional to the interaction strength, due to the gapless modes in the reservoirs, and a contribution quadratic in temperature, which originates from the incoherent tunneling of Bogoliubov quasiparticles. Importantly, the zero-temperature part dominates over the thermal component up to $T \sim \mu /\left(n a^{3}\right)^{1 / 4} \gg \mu$, where $\sqrt{n a^{3}}$ is the small parameter in the Bogoliubov theory.

We also find no contribution from the condensate to heat transport. As a result, the Lorenz number $L=\frac{L_{22}}{T L_{11}}-\frac{L_{12} L_{21}}{T L_{11}^{2}}$ and the Seebeck coefficient $S=\frac{L_{12}}{L_{11}}$, which characterize the relation between entropy and particle currents, go to 0 as the temperature is reduced. The leading temperature dependence turns out to be quadratic and cubic, respectively, for the Lorenz number and Seebeck coefficient, again a consequence of the phonon Green's function structure (see Appendix B). We note that this behavior of $L$ is quite different from the Wiedemann-Franz law, where $L$ is given by a universal number. Normally, this law is obeyed when charge and entropy are carried by the same fundamental processes such as quasiparticles in Fermi liquids [46,48] and magnons in spin systems $[49,50]$. Even in a BEC at $T \approx T_{c}$, it is predicted due to dominant quasiparticle transport [47]. Remarkably, we find that even though mass and heat transport originates from quasiparticles, the presence of the anomalous tunneling process yields a large violation of the Widemann-Franz law.

Figure 4 illustrates these results, where the conductance [in Fig. 4(a)] and the Seebeck coefficient and Lorenz numbers [in Fig. 4(b)] are presented as a function of $T / \mu$. Over the whole range of temperatures, the results are dominated by the zero-temperature contribution to the current originating from the phonon contribution. Note that the Bogoliubov theory fails around the critical temperature of a BEC, so we cannot extrapolate our results to the high-temperature regime covered, in particular, in [47].

\section{EXPERIMENTAL CONSIDERATIONS}

In this section, we discuss the situation of finite reservoirs yielding a finite charging energy, which is relevant to coldatom experiments.

\section{A. Time evolution of thermodynamic quantities}

Quantum gas two-terminal or double-well setups, to which our model directly applies, feature finite-sized reservoirs with a chemical potential self-consistently determined from the atom number. As a result, the coupling between the reservoirs competes with their finite compressibility, yielding the crossover between Josephson oscillations and nonlinear selftrapping [51]. The presence of a finite DC conductance implies directly that the self-trapping regime is not stable, as a system initialized at a large bias will slowly relax into the low-bias, Josephson oscillation regime. Such a relaxation has been reported in [52] and [53].

We now consider a similar scenario for single-channel point-contact transport accounting explicitly for the effects of finite-sized reservoirs. As mentioned before, we fix the parameters $\left(\mu=400 \mathrm{nK}, a=25 \mathrm{~nm}\right.$, and $\left.n=10^{19} \mathrm{~m}^{-3}\right)$. For such a parameter choice with $t \rho(\mu) \sim 0.1$, the ratio of the condensation energy to the Josephson coupling energy turns out to be of the order of $10^{6}$. For a typical initial bias of $\Delta N / N \sim 0.1$ [54], expressed in terms of the particle number relative imbalance, the system is initially deeply in the selftrapping regime $[33,51]$.

We start with a description of the thermodynamics of the reservoirs at equilibrium. The differences in thermodynamic quantities between the reservoirs obey the Maxwell relations

$$
\left(\begin{array}{c}
\Delta N \\
\Delta S
\end{array}\right)=\left(\begin{array}{ll}
\kappa & \alpha \\
\alpha & \frac{C_{\mu}}{T}
\end{array}\right)\left(\begin{array}{c}
\Delta \mu \\
\Delta T
\end{array}\right),
$$

where $\Delta N, \Delta S, \kappa, \alpha$, and $C_{\mu}$ are the relative atom-number difference, relative entropy difference, compressibility, dilatation coefficient, and heat capacity at constant $\mu$, respectively. We note that the thermodynamic coefficients $\left(\kappa, \alpha\right.$, and $\left.C_{\mu}\right)$ are sensitive to the geometry of the reservoirs. In a box-type reservoir, they are calculated with the Bogoliubov theory as

$$
\begin{gathered}
\kappa_{b} \approx \frac{\Omega}{g}+\frac{\pi^{2} \Omega T^{4}}{24 c^{3} \mu^{2}}, \\
\alpha_{b} \approx-\frac{\pi^{2} \Omega T^{3}}{15 c^{3} \mu}, \\
C_{\mu, b} \approx \frac{2 \pi^{2} \Omega T^{3}}{15 c^{3}},
\end{gathered}
$$


where $\Omega$ is the volume in each reservoir. In contrast, in a harmonically trapped reservoir, we obtain

$$
\begin{gathered}
\kappa_{h} \approx \frac{\Omega}{g}-\frac{0.36 \Omega T^{7 / 2}}{\pi c^{3} \mu^{3 / 2}}, \\
\alpha_{h} \approx \frac{2.5 \Omega T^{5 / 2}}{\pi c^{3} \mu^{1 / 2}}, \\
C_{\mu, h} \approx \frac{13 \Omega \mu^{1 / 2} T^{5 / 2}}{\pi c^{3}},
\end{gathered}
$$

where we assume that the trap is isotropic, in which case $\Omega=\frac{4 \pi}{3}\left(\frac{2 \mu}{M \omega_{\mathrm{ho}}^{2}}\right)^{3 / 2}$ with the trapping frequency $\omega_{\mathrm{ho}}$. The temperature and chemical potential responses to variations of the internal energy and particle number depend on the shape of the reservoirs and are, thus, different for box and harmonic traps. As we show below, this causes different behaviors of the time evolutions of thermodynamic quantities.

We now describe the slow time evolution of the thermodynamic quantities characterizing the reservoirs resulting from quasi-steady-state currents. The quasi-steady-state approximation, well verified in the experimentally relevant situations where DC transport is concerned, implies that the reservoirs are at thermal equilibrium at each point in time [6]. This allows for a direct generalization of the linear response results reported above to the case of slow time evolution of biases.

To deal with the case where biases between the reservoirs can evolve slowly in time, we adopt the model in which $\phi(\tau)$ is an independent dynamical variable [20,52]. There, in addition to Eq. (8), we consider the relations

$$
\begin{gathered}
\frac{1}{2} \frac{d \Delta N}{d \tau}=-I_{1}, \\
\frac{T}{2} \frac{d \Delta S}{d \tau}=-I_{2}, \\
\frac{d \phi}{d \tau}=\Delta \mu,
\end{gathered}
$$

which represent Kirchhoff's law, applicable in the lowfrequency regime consistent with the quasi-steady-state hypothesis. By numerically solving Eqs. (8) and (15)-(17), the time evolution of the thermodynamic quantities can be determined.

To discuss the global structure of the time evolution, we also introduce an approximation that neglects the AC components of the currents. In this case, by combining Eq. (7) and Eq. (8), we obtain

$$
\tau_{0} \frac{d}{d \tau}\left(\begin{array}{c}
\Delta N / \kappa \\
\Delta T
\end{array}\right)=-\left(\begin{array}{cc}
1 & -S_{\text {eff }} \\
-\frac{S_{\text {eff }}}{l} & \frac{L+S_{\text {eff }}^{2}}{l}
\end{array}\right)\left(\begin{array}{c}
\Delta N / \kappa \\
\Delta T
\end{array}\right),
$$

where $\tau_{0}=\kappa /\left(2 L_{11}\right)$ is the transport time for mass transport, $l \equiv C_{\mu} /(\kappa T)-(\alpha / \kappa)^{2}$ is the analog of the Lorenz number for the reservoirs, and $S_{\text {eff }} \equiv \alpha / \kappa-S$ is the effective Seebeck coefficient. Since the equation above is the first-order differential equation, the time evolutions can analytically be determined. The general solution is expressed as

$$
\begin{aligned}
& \Delta N(\tau)=\frac{1}{2}\left\{e^{-\tau / \tau_{-}}+e^{-\tau / \tau_{+}}-\left(1-\frac{L+S_{\mathrm{eff}}^{2}}{l}\right) \frac{e^{-\tau / \tau_{-}}-e^{-\tau / \tau_{+}}}{\lambda_{+}-\lambda_{-}}\right\} \Delta N(0)+\frac{S_{\mathrm{eff}} \kappa\left(e^{-\tau / \tau_{-}}-e^{-\tau / \tau_{+}}\right)}{\lambda_{+}-\lambda_{-}} \Delta T(0), \\
& \Delta T(\tau)=\frac{1}{2}\left\{e^{-\tau / \tau_{-}}+e^{-\tau / \tau_{+}}-\left(1-\frac{L+S_{\mathrm{eff}}^{2}}{l}\right) \frac{e^{-\tau / \tau_{-}}-e^{-\tau / \tau_{+}}}{\lambda_{+}-\lambda_{-}}\right\} \Delta T(0)+\frac{S_{\mathrm{eff}}\left(e^{-\tau / \tau_{-}}-e^{-\tau / \tau_{+}}\right)}{l \kappa\left(\lambda_{+}-\lambda_{-}\right)} \Delta N(0) .
\end{aligned}
$$

Here, the decay-time parameters $\tau_{ \pm}$are expressed as

$$
\tau_{ \pm}=\tau_{0} / \lambda_{ \pm}
$$

with

$$
\lambda_{ \pm}=\frac{1}{2}\left(1+\frac{L+S_{\mathrm{eff}}^{2}}{l}\right) \pm \sqrt{\frac{S_{\mathrm{eff}}^{2}}{l}+\frac{1}{4}\left(1-\frac{L+S_{\mathrm{eff}}^{2}}{l}\right)^{2}} .
$$

In Fig. 5 we present this result for the initial conditions $\Delta N(0) / N=0.2$ and $\Delta T(0) / T=0.2$, together with a numerical solution including the AC components. The parameters mentioned before are used with $T=150 \mathrm{nK}$ and $t \rho(\mu)=$ 0.2 . Under these parameters, regardless of trapped geometries, we obtain $\tau_{0} \sim 10 \mathrm{~s}$, similar to the case of a single-mode conductor for fermionic atoms [6]. The large conductance of the $\mathrm{BEC}$ compared with one expected for noninteracting fermions is compensated by the much larger compressibility of the reservoirs, yielding similar time scales. This illustrates the important role of the finite-size reservoirs in the interpretation of the experiments.

In the case of harmonically trapped reservoirs, the initial relative atom number difference decays on the time scale $\tau_{0}$ as shown in Fig. 5(a). However, the low values of the Lorenz number and Seebeck coefficient at low temperature and the near-cancellation of the effective Seebeck coefficient between the QPC and the reservoir contributions [55] lead to the slower decay of the initial temperature difference. In the case of box reservoirs [37,56,57], on the other hand, both $\Delta N$ and $\Delta T$ decay on the time scale $\tau_{0}$ despite the low values of the Lorenz number and Seebeck coefficient. The faster decay of $\Delta T$ in box reservoirs is due to the fact that $L / l \approx 0.5$, that is, the small Lorenz number is compensated by the low value of the analog of the Lorenz number for reservoirs. Finally, we note that at large times where $\tau / \tau_{0} \gg 1$ and $\Delta N / N \approx$ $\Delta T / T \approx 0$, the effect of the AC oscillations can be seen in the numerical calculations. Except for this effect, the overall differences between the analytic solutions (dashed curves) and the numerical solutions with the AC components (solid curves) are minuscule.

\section{B. Shapiro resonance as a probe of AC components}

The effects of the phonon modes on the AC currents could be observed by direct Fourier analysis of the reservoirs' population dynamics. This has been demonstrated at low frequencies for wide junctions [23,24] but might prove 


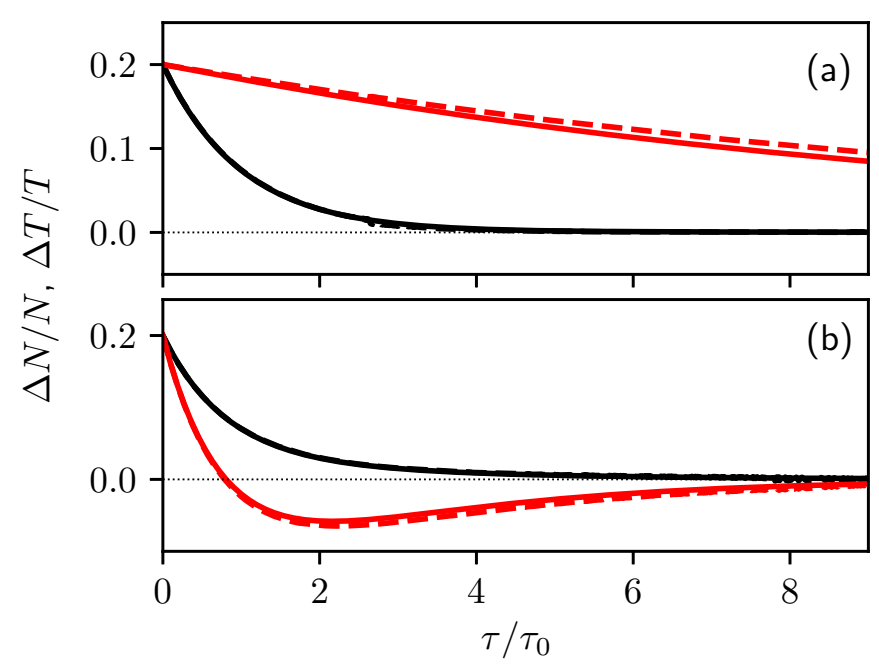

FIG. 5. Time evolutions of $\Delta N(\tau) / N$ (black lines) and $\Delta T(\tau) / T$ (red lines), for initial conditions $\Delta N(0) / N=0.2$ and $\Delta T(0) / T=$ 0.2 . The reservoirs are (a) harmonic traps and (b) box traps. Solid lines describe the full numerical solutions and dashed lines show the results of Eqs. (19) and (20).

difficult for weak harmonics at high frequencies. Therefore, we propose to circumvent this difficulty by leveraging the time-dependent control over trapping potentials and observing Shapiro resonances [58,59], mapping the harmonics of the Josephson current onto the DC component.

To this end, we consider the time-dependent hopping amplitude such that the phase parameter is given by

$$
\phi(\tau)=\phi_{0}+\Delta \mu \tau+\alpha \sin \left(\omega_{1} \tau\right),
$$

where $\omega_{1}$ and $\alpha$ are the oscillation frequency and its amplitude, respectively. By substituting the above into the particle current expression, we obtain

$$
\begin{aligned}
I_{1}(\tau)= & \sum_{m=0}^{\infty}\left[I_{m}^{\mathrm{nd}} \sin (m \phi(\tau))+I_{m}^{\mathrm{d}} \cos (m \phi(\tau))\right] \\
= & \sum_{m=0}^{\infty} I_{m}^{\mathrm{nd}}\left[\sin \left(m\left(\phi_{0}+\Delta \mu \tau\right)\right) \cos \left(m \alpha \sin \left(\omega_{1} \tau\right)\right)\right. \\
& +\cos \left(m\left(\phi_{0}+\Delta \mu \tau\right)\right) \sin \left(m \alpha \sin \left(\omega_{1} \tau\right)\right] \\
& +\sum_{m=0}^{\infty} I_{m}^{\mathrm{d}}\left[\cos \left(m\left(\phi_{0}+\Delta \mu \tau\right)\right) \cos \left(m \alpha \sin \left(\omega_{1} \tau\right)\right)\right. \\
& -\sin \left(m\left(\phi_{0}+\Delta \mu \tau\right)\right) \sin \left(m \alpha \sin \left(\omega_{1} \tau\right)\right],
\end{aligned}
$$

which can be expressed in terms of the Bessel functions of order $n J_{n}$, as

$$
\begin{aligned}
I_{1}(\tau)= & \sum_{m, n=0}^{\infty} J_{n}(m \alpha)\left[I_{m}^{\mathrm{nd}} \sin \left(m \phi_{0}+m \Delta \mu \tau-n \omega_{1} \tau\right)\right. \\
& \left.+(-1)^{n} I_{m}^{\mathrm{d}} \cos \left(m \phi_{0}+m \Delta \mu \tau-n \omega_{1} \tau\right)\right] \\
& +\sum_{m=0}^{\infty} \sum_{n=1}^{\infty} J_{n}(m \alpha)\left[I_{m}^{\mathrm{nd}} \sin \left(m \phi_{0}+m \Delta \mu \tau+n \omega_{1} \tau\right)\right. \\
& \left.+I_{m}^{\mathrm{d}} \cos \left(m \phi_{0}+m \Delta \mu \tau+n \omega_{1} \tau\right)\right]
\end{aligned}
$$

In the above, the second line on the right-hand side always depends on time under $\Delta \mu, \omega_{1}>0$ and, therefore, is typically averaged out in two-terminal setups of ultracold atomic gases. However, the first line becomes time independent and gives a nonzero DC contribution if the following condition is satisfied:

$$
\Delta \mu=\frac{n}{m} \omega_{1}
$$

This is the condition under which the Shapiro resonance occurs. For instance, if

$$
\Delta \mu=\frac{\omega_{1}}{3},
$$

third-order harmonics of the AC current are dominantly contributing to the DC current, since the lower harmonics are averaged out.

\section{CONCLUDING REMARKS}

Our work elucidates the relation between the dissipative current and the superfluid character of the BEC. The QPC implements a coherent coupling of the superfluid component with the phonon modes, in the spirit of the celebrated Landau argument on superfluidity. Even though the zero-temperature DC component does not carry any entropy, as demonstrated by the zero value of the Seebeck coefficient, it cannot be identified with a superfluid current. This also illustrates the difficulty in modeling the dynamics of superfluids in mesoscopic structures using effective two-fluid models: in particular, QPCs behave very differently than superleaks in helium, and the fountain effect is not expected [60].

Charge-neutral fermionic superfluids also feature a gapless mode resulting from the $\mathrm{U}(1)$ symmetry breaking. While we cannot extrapolate the bosonic results derived here to the fermionic case [61], Bogoliubov theory is adequate for the far molecular side of the BEC-BCS crossover. The $1 / g$ dependence of the zero-temperature contribution suggests that it should become less relevant as the system approaches the Feshbach resonance. This is confirmed by the good agreement between low-temperature transport experiments at unitarity and multiple Andreev reflection theory observed in [44].

A direct generalization of our theory could describe spin transport in spinor BECs or include spin-orbit coupling in the reservoirs and the contact. It also predicts coherent heat current oscillations at $2 \Delta \mu$, encountered in superconductors [62] but not studied so far with cold atoms, as well as the current noise spectrum. While current noise in the two-terminal setup has never been measured in quantum gas experiments, we expect it to become accessible in future generations of experimental setups [63].

\section{ACKNOWLEDGMENTS}

We thank Thierry Giamarchi and Martin Lebrat for careful reading of the manuscript and discussions and, also, thank Masahito Ueda for discussions. S.U. was supported by JSPS KAKENHI Grant No. JP17K14366, the Matsuo Foundation, and a Waseda University Grant for Special Research Projects 
(No. 2019C-461). J.-P.B. acknowledges funding from the European Research Council under the European Union's Horizon 2020 research and innovation program (Grant Agree- ment No. 714309), the Sandoz Family Foundation-Monique de Meuron program for Academic Promotion, the SNSF (Project No. 184654), and the EPFL.

\section{APPENDIX A: CURRENT EXPRESSION}

Here we provide some details on the derivation of the current formula [Eqs. (2)-(4)] underlying the results of this work. The notations are those used in the text. Using Heisenberg equations of motion, the particle and heat currents in the tunneling Hamiltonian can be expressed as

$$
\begin{gathered}
I_{1}(\tau)=-\frac{i t}{\Omega} \sum_{\mathbf{p}, \mathbf{k}}\left\langle b_{\mathbf{p}, R}^{\dagger}(\tau) b_{\mathbf{k}, L}(\tau)-b_{\mathbf{k}, L}^{\dagger}(\tau) b_{\mathbf{p}, R}(\tau)\right\rangle, \\
I_{2}(\tau)=\frac{t}{\Omega} \sum_{\mathbf{p}, \mathbf{k}}\left\langle b_{\mathbf{p}, R}^{\dagger}(\tau) \frac{d}{d \tau} b_{\mathbf{k}, L}(\tau)+\frac{d}{d \tau} b_{\mathbf{k}, L}^{\dagger}(\tau) b_{\mathbf{p}, R}(\tau)\right\rangle,
\end{gathered}
$$

where the field operator in the momentum space satisfies $\psi_{L(R)}(\mathbf{x})=\frac{1}{\sqrt{\Omega}} \sum_{\mathbf{k}} e^{i \mathbf{k} \cdot \mathbf{x}} b_{\mathbf{k}, L(R)}$, and $\langle\ldots\rangle$ is the average under the total Hamiltonian with the tunneling term. To go further, we rewrite the above expression as

$$
\begin{gathered}
I_{1}(\tau)=2 \operatorname{Re}\left[\hat{G}_{L R}^{<}(\tau, \tau) \hat{t}(\tau)\right]_{11}, \\
I_{2}(\tau)=2 \lim _{\tau^{\prime} \rightarrow \tau} \frac{d}{d \tau} \operatorname{Re}\left[i \hat{G}_{L R}^{<}\left(\tau, \tau^{\prime}\right) \hat{t}\left(\tau^{\prime}\right)\right]_{11},
\end{gathered}
$$

where

$$
\begin{gathered}
\hat{G}_{L R}^{<}\left(\tau, \tau^{\prime}\right)=-\frac{i}{\Omega} \sum_{\mathbf{p}, \mathbf{k}}\left(\begin{array}{cc}
\left\langle b_{\mathbf{p}, R}^{\dagger}\left(\tau^{\prime}\right) b_{\mathbf{k}, L}(\tau)\right\rangle & \left\langle b_{\mathbf{p}, R}\left(\tau^{\prime}\right) b_{\mathbf{k}, L}(\tau)\right\rangle \\
\left\langle b_{\mathbf{p}, R}^{\dagger}\left(\tau^{\prime}\right) b_{\mathbf{k}, L}^{\dagger}(\tau)\right\rangle & \left\langle b_{\mathbf{p}, R}\left(\tau^{\prime}\right) b_{\mathbf{k}, L}^{\dagger}(\tau)\right\rangle
\end{array}\right), \\
\hat{t}(\tau)=t\left(\begin{array}{cc}
e^{-i \phi(\tau)} & 0 \\
0 & e^{i \phi(\tau)}
\end{array}\right)
\end{gathered}
$$

and we perform the gauge transformation in the tunneling Hamiltonian [26,39], where the chemical potential difference between the reservoirs is reflected in the phase factor of the tunneling amplitude. Since it is difficult to evaluate the nonequilibrium average $\langle\ldots\rangle$ directly, we perform the expansion in $t$ by means of the Keldysh formalism, which allows us to calculate the currents in terms of Green's functions at equilibrium. Indeed, by using the so-called Langreth rules [34], we can obtain the Dyson equation

$$
\hat{G}^{<}=\left(\hat{1}+\hat{G}^{R} \circ \hat{V}\right) \circ \hat{g}^{<} \circ\left(\hat{1}+\hat{V} \circ \hat{G}^{A}\right),
$$

where $\hat{1}=\operatorname{diag}(1,1) \delta\left(\tau-\tau^{\prime}\right), \hat{V}$ represents $\hat{t}$ or $\hat{t}^{\dagger}$, and $\hat{G}^{R(A)}$ is the coupled retarded (advanced) Green's function obeying

$$
\hat{G}^{R(A)}=\hat{g}^{R(A)}+\hat{g}^{R(A)} \circ \hat{V} \circ \hat{G}^{R(A)} .
$$

Here, we introduce the uncoupled retarded (advanced) Green's function $\hat{g}^{R(A)}$, which is evaluated at equilibrium. By using the low-frequency expansions in the Bogoliubov theory, this is expressed in frequency space as

$$
\begin{aligned}
\hat{g}_{\alpha \beta}^{R}(\omega) & =-\frac{i}{\Omega} \sum_{\mathbf{k}} \int_{-\infty}^{\infty} d \tau e^{i \omega \tau} \theta(\tau)\left(\begin{array}{cc}
\left\langle\left[b_{\mathbf{k}, \alpha}(\tau), b_{\mathbf{k}, \beta}^{\dagger}(0)\right]\right\rangle_{0} & \left\langle\left[b_{\mathbf{k}, \alpha}(\tau), b_{-\mathbf{k}, \beta}(0)\right]\right\rangle_{0} \\
\left\langle\left[b_{-\mathbf{k}, \alpha}^{\dagger}(\tau), b_{\mathbf{k}, \beta}^{\dagger}(0)\right]\right\rangle_{0} & \left\langle\left[b_{\mathbf{k}, \alpha}^{\dagger}(\tau), b_{\mathbf{k}, \beta}(0)\right]\right\rangle_{0}
\end{array}\right) \\
& \approx \delta_{\alpha, \beta}\left(\begin{array}{cc}
-\frac{\mu_{\alpha} \omega_{c}}{2 \pi^{2} c^{3}}-\frac{\omega_{c} \omega}{2 \pi^{2} c_{\alpha}^{3}}+\frac{\mu_{\alpha} \omega^{2}}{2 \pi^{2} \omega_{c} c_{\alpha}^{3}}-i\left[\frac{\mu_{\alpha} \omega}{4 \pi c_{\alpha}^{3}}+\frac{\omega^{2}}{4 \pi c_{\alpha}^{3}}\right] & \frac{\mu_{\alpha} \omega_{c}}{2 \pi^{2} c_{\alpha}^{3}}-\frac{\mu_{\alpha} \omega^{2}}{2 \pi^{2} \omega_{c} c_{\alpha}^{3}}+i \frac{\mu_{\alpha} \omega}{4 \pi c_{\alpha}^{3}} \\
\frac{\mu_{\alpha} \omega_{c}}{2 \pi^{2} c_{\alpha}^{3}}-\frac{\mu_{\alpha} \omega^{2}}{2 \pi^{2} \omega_{c} c_{\alpha}^{3}}+i \frac{\mu_{\alpha} \omega}{4 \pi c_{\alpha}^{3}} & \left.-\frac{\mu_{\alpha} \omega_{c}}{2 \pi^{2} c_{\alpha}^{3}}+\frac{\omega_{c} \omega}{2 \pi^{2} c_{\alpha}^{3}}+\frac{\mu_{\alpha} \omega^{2}}{2 \pi^{2} \omega_{c} c_{\alpha}^{3}}+i\left[-\frac{\mu_{\alpha} \omega}{4 \pi c_{\alpha}^{3}}+\frac{\omega^{2}}{4 \pi c_{\alpha}^{3}}\right]\right)
\end{array}\right.
\end{aligned}
$$

where $\alpha, \beta=\mathrm{L}$ or $\mathrm{R}, \hat{g}^{A}(\omega)=\left[\hat{g}^{R}(\omega)\right]^{\dagger}$, and $\omega_{c}$ is the cutoff frequency of the tunneling Hamiltonian and is chosen as the average chemical potential [43]. Similarly, the uncoupled lesser Green's function in frequency space is obtained as

$$
\begin{aligned}
& \hat{g}_{\alpha \beta}^{<}(\omega)=-\frac{i}{\Omega} \sum_{\mathbf{k}} \int_{-\infty}^{\infty} d \tau e^{i \omega \tau}\left(\begin{array}{cc}
\left\langle b_{\mathbf{k}, \beta}^{\dagger}(0) b_{\mathbf{k}, \alpha}(\tau)\right\rangle_{0} & \left\langle b_{-\mathbf{k}, \beta}(0) b_{\mathbf{k}, \alpha}(\tau)\right\rangle_{0} \\
\left\langle b_{\mathbf{k}, \beta}^{\dagger}(0) b_{-\mathbf{k}, \alpha}^{\dagger}(\tau)\right\rangle_{0} & \left\langle b_{\mathbf{k}, \beta}(0) b_{\mathbf{k}, \alpha}^{\dagger}(\tau)\right\rangle_{0}
\end{array}\right) \\
& \approx-\frac{2 \pi i \sqrt{\mu_{\alpha} \mu_{\beta}}}{g} \delta(\omega)\left(\begin{array}{ll}
1 & 1 \\
1 & 1
\end{array}\right)+i \delta_{\alpha, \beta} n_{\alpha}(\omega)\left(\begin{array}{cc}
-\left[\frac{\mu_{\alpha} \omega}{2 \pi c_{\alpha}^{3}}+\frac{\omega^{2}}{2 \pi c_{\alpha}^{3}}\right] & \frac{\mu_{\alpha} \omega}{2 \pi c_{\alpha}^{3}} \\
\frac{\mu_{\alpha} \omega}{2 \pi c_{\alpha}^{3}} & -\left[\frac{\mu_{\alpha} \omega}{2 \pi c_{\alpha}^{3}}-\frac{\omega^{2}}{2 \pi c_{\alpha}^{3}}\right]
\end{array}\right),
\end{aligned}
$$


where

$$
n_{\alpha}(\omega)=\frac{1}{e^{\omega / T_{\alpha}}-1}
$$

is the Bose distribution function. The peculiar feature of the lesser Green's function above is the presence of the contribution from BECs corresponding to the first term on the right-hand side of Eq. (10). This term is present even if $\alpha \neq \beta$, which leads to the presence of odd-order harmonics in the current. Indeed, by using uncoupled Green's functions, we obtain

$$
\begin{aligned}
& \hat{G}_{L R}^{<\text {,even }}=\left(\hat{\imath}+\hat{G}_{L R}^{R} \hat{t}\right) \circ \hat{g}_{L L}^{<} \circ \hat{t}^{\dagger} \hat{G}_{R R}^{A}+\hat{G}_{L L}^{R} \hat{t}^{\dagger} \circ \hat{g}_{R R}^{<} \circ\left(\hat{\imath}+\hat{t} \hat{G}_{L R}^{A}\right), \\
& \hat{G}_{L R}^{<, \text {odd }}=\left(\hat{\imath}+\hat{G}_{L R}^{R} \hat{t}\right) \circ \hat{g}_{L R}^{<} \circ\left(\hat{\imath}+\hat{t} \hat{G}_{L R}^{A}\right)+\hat{G}_{L L}^{R} \hat{t}^{\dagger} \circ \hat{g}_{R L}^{<} \circ \hat{t}^{\dagger} \hat{G}_{R R}^{A},
\end{aligned}
$$

where the superscript "even" ("odd") represents the components of even (odd) harmonics in the current. As is clear from the expressions above, $\hat{G}_{L R}^{<, \text {odd }}=\hat{0}$, since $\hat{g}_{L R}^{<}=\hat{g}_{R L}^{<}=\hat{0}$ in the fermionic system.

For efficient numerics, as in the case of superconductors, it is convenient to introduce the renormalized tunneling matrix, (5). By using this renormalized tunneling matrix, the current formula shown in Sec. II can be obtained.

\section{APPENDIX B: CURRENT EXPRESSIONS UP TO THE LINEAR RESPONSE}

We now derive several current expressions up to the linear response theory discussed in Sec. III. In this case, the substitution $\hat{T}^{R(A)} \rightarrow \hat{t}$ is allowed, and analytic expressions are obtained.

We first examine the current components linear in $t$. The presence of such components allows nonzero $I_{q}^{\text {odd }}(\tau)$. By substituting $\hat{T}^{R(A)} \rightarrow \hat{t}$ into Eq. (3), we obtain

$$
\begin{gathered}
I_{1,1}=0, \\
I_{1,-1}=-\frac{2 i t \sqrt{\mu_{L} \mu_{R}}}{g} .
\end{gathered}
$$

Thus, the particle current up to $t$ is given by $I_{1}(\tau)=-\frac{2 t \sqrt{\mu_{L} \mu_{R}}}{g} \sin \phi(\tau)$. A similar calculation is allowed for the heat current. It is then straightforward to show $I_{2}(\tau)=0$, that is, absence of the Josephson heat current up to $t$.

We next examine the current components quadratic in $t$, which arise from $I_{q}^{\text {even }}(\tau)$ and include both DC and AC parts. The DC part of the particle current is expressed as

$$
\begin{aligned}
I_{1,0} & =\int_{-\infty}^{\infty} \frac{t^{2} d \omega}{\pi} \operatorname{Re}\left[\hat{g}_{L L, 11}^{R}(\omega) \hat{g}_{R R, 11}^{<}(\omega+\Delta \mu)+\hat{g}_{L L, 11}^{<}(\omega) \hat{g}_{R R, 11}^{A}(\omega+\Delta \mu)\right] \\
& =I_{1,0}^{c}+I_{1,0}^{b},
\end{aligned}
$$

where we introduce

$$
\begin{aligned}
& I_{1,0}^{c}=\frac{t^{2}}{g}\left[\mu_{R} \rho_{L}(-\Delta \mu)-\mu_{L} \rho_{R}(\Delta \mu)\right], \\
& I_{1,0}^{b}=t^{2} \int_{-\infty}^{\infty} \frac{d \omega}{2 \pi} \rho_{L}(\omega) \rho_{R}(\omega+\Delta \mu)\left[n_{L}(\omega)-n_{R}(\omega+\Delta \mu)\right],
\end{aligned}
$$

with the density of state

$$
\rho_{\alpha}(\omega)=-2 \operatorname{Im}\left[\hat{g}_{\alpha \alpha, 11}^{R}(\omega)\right] .
$$

Physically, $I_{1,0}^{c}$ describes the contribution from the conversion process between condensate and phonon, and $I_{1,0}^{b}$ describes the contribution from the tunneling process of phonons, which is comparable to the tunneling current formula in fermionic quasiparticles [39]. Based on the above expressions, we next look at the regime up to linear in $\Delta \mu$ and $\Delta T$ and determine the transport coefficients in Onsager's matrix. By using Eq. (A11), $I_{1,0}^{c}$ is easily obtained as

$$
I_{1,0}^{c} \approx \frac{t^{2} \mu^{2}}{\pi c^{3} g} \Delta \mu
$$

where we introduce the average chemical potential $\mu$ and average sound velocity $c$. On the other hand, by using

$$
n_{L}(\omega)-n_{R}(\omega+\Delta \mu) \approx \frac{\partial n(\omega)}{\partial \omega} \Delta \mu+\frac{\partial n(\omega)}{\partial T} \Delta T=\frac{1}{4 T} \frac{\Delta \mu}{\sinh ^{2}(\omega /(2 T))}+\frac{\omega}{4 T^{2}} \frac{\Delta T}{\sinh ^{2}(\omega /(2 T))},
$$

with the average temperature $T, I_{1,0}^{b}$ is obtained as

$$
I_{1,0}^{b} \approx L_{11}^{b} \Delta \mu+L_{12} \Delta T,
$$


where

$$
\begin{gathered}
L_{11}^{b}=\frac{t^{2}}{4 T} \int_{-\infty}^{\infty} \frac{d \omega}{2 \pi} \frac{\rho_{L}(\omega) \rho_{R}(\omega)}{\sinh ^{2}(\omega /(2 T))} \approx \frac{t^{2} \mu^{2} T^{2}}{4 \pi^{3} c^{6}} \int_{-\infty}^{\infty} d x \frac{x^{2}}{\sinh ^{2} x}=\frac{t^{2} \mu^{2} T^{2}}{12 \pi c^{6}}, \\
L_{12}=\frac{t^{2}}{4 T^{2}} \int_{-\infty}^{\infty} \frac{d \omega}{2 \pi} \frac{\omega \rho_{L}(\omega) \rho_{R}(\omega)}{\sinh ^{2}(\omega /(2 T))} \approx \frac{2 t^{2} \mu T^{3}}{\pi^{3} c^{6}} \int_{-\infty}^{\infty} d x \frac{x^{4}}{\sinh ^{2} x}=\frac{2 \pi t^{2} \mu T^{3}}{15 c^{6}} .
\end{gathered}
$$

To obtain the above, we only keep the contributions with the leading temperature dependence. In $L_{11}^{b}$, this contribution arises from a term $\propto \int_{-\infty}^{\infty} \frac{d \omega}{2 \pi} \frac{\omega^{2}}{\sinh ^{2}(\omega /(2 T))}$. Similarly, the leading-order contribution in $L_{12}$ arises from a term $\propto \int_{-\infty}^{\infty} \frac{d \omega}{2 \pi} \frac{\omega^{4}}{\sinh ^{2}(\omega /(2 T))}[64]$.

In addition, $L_{22}$ can be obtained from the DC part of the heat current at $\Delta \mu=0$, which is given by

$$
\begin{aligned}
I_{2,0} & =\int_{-\infty}^{\infty} \frac{t^{2} \omega d \omega}{\pi} \operatorname{Re}\left[\hat{g}_{L L, 11}^{R}(\omega) \hat{g}_{R R, 11}^{<}(\omega)+\hat{g}_{L L, 11}^{<}(\omega) \hat{g}_{R R, 11}^{A}(\omega)\right] \\
& =t^{2} \int_{-\infty}^{\infty} \frac{d \omega}{2 \pi} \omega \rho_{L}(\omega) \rho_{R}(\omega)\left[n_{L}(\omega)-n_{R}(\omega)\right] \approx L_{22} \Delta T .
\end{aligned}
$$

As in the case of $L_{11}^{b}$ and $L_{12}, L_{22}$ can be obtained as

$$
L_{22} \approx \frac{t^{2} \mu^{2} T^{3}}{\pi^{3} c^{6}} \int_{-\infty}^{\infty} d x \frac{x^{4}}{\sinh ^{2} x}=\frac{\pi t^{2} \mu^{2} T^{3}}{15 c^{6}} .
$$

Calculation of the AC components is done in a similar manner. At zero temperature, the AC components in the particle current are obtained as

$$
\begin{gathered}
I_{1,2}=0, \\
I_{1,-2}=-\frac{2 i \mu_{R} t^{2}}{g} \hat{g}_{L L, 12}^{R}(\Delta \mu)-\frac{2 i \mu_{L} t^{2}}{g} \hat{g}_{R R, 12}^{A}(-\Delta \mu) .
\end{gathered}
$$

Therefore, in the low-bias regime, the harmonics proportional to $e^{ \pm i 2 \phi(\tau)}$ are approximated as

$$
\frac{t^{2} \mu^{2} \Delta \mu}{\pi g c^{3}} \cos (2 \phi(\tau))-\frac{2 t^{2} \mu^{3}}{\pi g c^{3}} \sin (2 \phi(\tau)) .
$$

The result above shows that the AC components quadratic in $t$ yield both dissipative and nondissipative terms. In addition, at a low bias, the amplitude of the dissipative term depends on $\Delta \mu$ and that of the nondissipative term is independent of $\Delta \mu$.

[1] Y. V. Nazarov and Y. M. Blanter, Quantum Transport: Introduction to Nanoscience (Cambridge University Press, Cambridge, UK, 2009).

[2] S. Datta, Electronic Transport in Mesoscopic Systems (Cambridge University Press, Cambridge, UK, 1997).

[3] Y. M. Blanter and M. Büttiker, Phys. Rep. 336, 1 (2000).

[4] M. Esposito, U. Harbola, and S. Mukamel, Rev. Mod. Phys. 81, 1665 (2009).

[5] C.-C. Chien, S. Peotta, and M. Di Ventra, Nat. Phys. 11, 998 (2015).

[6] S. Krinner, T. Esslinger, and J.-P. Brantut, J. Phys.: Condens. Matter 29, 343003 (2017).

[7] S. Krinner, D. Stadler, D. Husmann, J.-P. Brantut, and T. Esslinger, Nature 517, 64 (2015).

[8] B. T. Seaman, M. Krämer, D. Z. Anderson, and M. J. Holland, Phys. Rev. A 75, 023615 (2007).

[9] A. Ramanathan, K. C. Wright, S. R. Muniz, M. Zelan, W. T. Hill, C. J. Lobb, K. Helmerson, W. D. Phillips, and G. K. Campbell, Phys. Rev. Lett. 106, 130401 (2011).

[10] F. Jendrzejewski, S. Eckel, N. Murray, C. Lanier, M. Edwards, C. Lobb, and G. K. Campbell, Phys. Rev. Lett. 113, 045305 (2014).
[11] C. Ryu and M. G. Boshier, New J. Phys. 17, 092002 (2015).

[12] I. Bloch, J. Dalibard, and W. Zwerger, Rev. Mod. Phys. 80, 885 (2008).

[13] D. B. Gutman, Y. Gefen, and A. D. Mirlin, Phys. Rev. B 85, 125102 (2012).

[14] C.-C. Chien, M. Di Ventra, and M. Zwolak, Phys. Rev. A 90, 023624 (2014).

[15] A. R. Kolovsky, Z. Denis, and S. Wimberger, Phys. Rev. A 98, 043623 (2018).

[16] A. Altland and B. D. Simons, Condensed Matter Field Theory (Cambridge University Press, Cambridge, UK, 2010).

[17] N. Nagaosa, Quantum Field Theory in Condensed Matter Physics (Springer Science \& Business Media, Berlin, 2013).

[18] A. M. Zagoskin, Quantum Theory of Many-Body Systems (Springer, Berlin, 1998), Vol. 174.

[19] D. Averin and A. Bardas, Phys. Rev. Lett. 75, 1831 (1995).

[20] S. Eckel, J. G. Lee, F. Jendrzejewski, C. J. Lobb, G. K. Campbell, and W. T. Hill, Phys. Rev. A 93, 063619 (2016).

[21] P.-F. Duc, M. Savard, M. Petrescu, B. Rosenow, A. Del Maestro, and G. Gervais, Sci. Adv. 1, e1400222 (2015). 
[22] M. Albiez, R. Gati, J. Fölling, S. Hunsmann, M. Cristiani, and M. K. Oberthaler, Phys. Rev. Lett. 95, 010402 (2005).

[23] S. Levy, Nature (London) 449, 579 (2007).

[24] L. J. LeBlanc, A. B. Bardon, J. McKeever, M. H. T. Extavour, D. Jervis, J. H. Thywissen, F. Piazza, and A. Smerzi, Phys. Rev. Lett. 106, 025302 (2011).

[25] K. Xhani, E. Neri, L. Galantucci, F. Scazza, A. Burchianti, K.-L. Lee, C. Barenghi, A. Trombettoni, M. Inguscio, and M. Zaccanti, Phys. Rev. Lett. 124, 045301 (2020).

[26] J. C. Cuevas, A. Martín-Rodero, and A. L. Yeyati, Phys. Rev. B 54, 7366 (1996).

[27] C. J. Bolech and T. Giamarchi, Phys. Rev. Lett. 92, 127001 (2004).

[28] C. J. Bolech and T. Giamarchi, Phys. Rev. B 71, 024517 (2005).

[29] S. Uchino and M. Ueda, Phys. Rev. Lett. 118, 105303 (2017).

[30] J. Yao, B. Liu, M. Sun, and H. Zhai, Phys. Rev. A 98, 041601(R) (2018).

[31] X. Han, B. Liu, and J. Hu, Phys. Rev. A 100, 043604 (2019).

[32] J. O. Andersen, Rev. Mod. Phys. 76, 599 (2004).

[33] L. Pitaevskii and S. Stringari, Bose-Einstein Condensation and Superfluidity (Oxford University Press, New York, 2016), Vol. 164.

[34] J. Rammer, Quantum Field Theory of Non-equilibrium States (Cambridge University Press, Cambridge, UK, 2007).

[35] A. Kamenev, Field Theory of Non-equilibrium Systems (Cambridge University Press, Cambridge, UK, 2011).

[36] We note that transport coefficients depend on biases in nonohmic cases, where the Lorenz number cannot be expressed solely in terms of fundamental constants.

[37] A. L. Gaunt, T. F. Schmidutz, I. Gotlibovych, R. P. Smith, and Z. Hadzibabic, Phys. Rev. Lett. 110, 200406 (2013).

[38] K. K. Likharev, Rev. Mod. Phys. 51, 101 (1979).

[39] G. D. Mahan, Many-Particle Physics (Springer Science \& Business Media, Berlin, 2013).

[40] C. Berthod and T. Giamarchi, Phys. Rev. B 84, 155414 (2011).

[41] A. L. Fetter and J. D. Walecka, Quantum Theory of ManyParticle Systems (Courier Corp., North Chelmsford, MA, 2012).

[42] The c-number part of the field operator does not evolve in time and so is that of lesser Green's function [41].

[43] F. Meier and W. Zwerger, Phys. Rev. A 64, 033610 (2001).
[44] D. Husmann, S. Uchino, S. Krinner, M. Lebrat, T. Giamarchi, T. Esslinger, and J.-P. Brantut, Science 350, 1498 (2015).

[45] T. Giamarchi, Quantum Physics in One Dimension (Oxford University Press, New York, 2004).

[46] H. van Houten and C. W. J. Beenakker, Phys. Today 49(7), 22 (1996).

[47] D. J. Papoular, L. P. Pitaevskii, and S. Stringari, Phys. Rev. Lett. 113, 170601 (2014).

[48] N. W. Ashcroft and N. D. Mermin, Solid State Physics (Thomson Learning, Boston, MA, 1976).

[49] K. Nakata, P. Simon, and D. Loss, Phys. Rev. B 92, 134425 (2015).

[50] K. Nakata, Y. Ohnuma, and M. Matsuo, Phys. Rev. B 98, 094430 (2018).

[51] A. Smerzi, S. Fantoni, S. Giovanazzi, and S. R. Shenoy, Phys. Rev. Lett. 79, 4950 (1997).

[52] A. Burchianti, F. Scazza, A. Amico, G. Valtolina, J. A. Seman, C. Fort, M. Zaccanti, M. Inguscio, and G. Roati, Phys. Rev. Lett. 120, 025302 (2018).

[53] G. Gauthier, S. S. Szigeti, M. T. Reeves, M. Baker, T. A. Bell, H. Rubinsztein-Dunlop, M. J. Davis, and T. W. Neely, Phys. Rev. Lett. 123, 260402 (2019).

[54] In this case, the transition between Josephson oscillation and self-trapping regimes occurs when the ratio of the condensation energy to the Josephson coupling energy becomes 10 to $10^{2}$.

[55] C. Grenier, C. Kollath, and A. Georges, C. R. Physique 17, 1161 (2016).

[56] B. Mukherjee, Z. Yan, P. B. Patel, Z. Hadzibabic, T. Yefsah, J. Struck, and M. W. Zwierlein, Phys. Rev. Lett. 118, 123401 (2017).

[57] N. Luick, L. Sobirey, M. Bohlen, V. P. Singh, L. Mathey, T. Lompe, and H. Moritz, arXiv:1908.09776.

[58] A. Barone and G. Paterno, Physics and Applications of the Josephson Effect (Wiley, New York, 1982).

[59] M. Tinkham, Introduction to Superconductivity (Courier Corp., North Chelmsford, MA, 2004).

[60] T. Karpiuk, B. Grémaud, C. Miniatura, and M. Gajda, Phys. Rev. A 86, 033619 (2012).

[61] S. Uchino, arXiv:2003.08672 [cond-mat.quant-gas].

[62] A. Fornieri and F. Giazotto, Nat. Nanotechnol. 12, 944 (2017).

[63] S. Uchino, M. Ueda, and J.-P. Brantut, Phys. Rev. A 98, 063619 (2018).

[64] We note that $\int_{-\infty}^{\infty} \frac{d \omega}{2 \pi} \frac{\omega^{3}}{\sinh ^{2}(\omega /(2 T))}=0$. 